\title{
Congenital Conjoined Double Medial Meniscus: A Case Report
}

\author{
Ahmed $W^{1 *}$, Ullah SMA ${ }^{2}$, Sarwar Z $Z^{3}$, Rahman $\mathrm{PK}^{4}$
}

\author{
${ }^{1}$ Associate Professor, Orthopaedic Surgery, National Institute of Traumatology and Orthopaedic Rehabilitation (NITOR), Dhaka, Bangladesh \\ ${ }^{2}$ Medical officer, Dhaka Medical College Hospital, Dhaka, Bangladesh \\ ${ }^{3}$ Clinical Fellow, Sports Medicine and Arthroscopy, Dhaka, Bangladesh \\ ${ }^{4}$ Professor Department of Radiology and Imaging, Sheikh Hasina National Institute of Burn and Plastic Surgery, Dhaka, Bangladesh
}

DOI: $\underline{10.36347 / \text { sjmcr.2020.v08i12.009 }}$

| Received: 05.12.2020 | Accepted: 16.12.2020 | Published: 22.12.2020

*Corresponding author: Ahmed $\mathrm{W}$

Abstract

Introduction: We report on a case of congenital conjoined double medial meniscus, which was incidentally found during arthroscopic anterior cruciate ligament reconstruction of a young patient. Both the meniscus were connected to each other throughout the whole length conjoined with having a junctional cleavage. And its periphery was connected to the joint capsule. This case demonstrates an interesting and very rare anatomical abnormality of the medial meniscus. We report on the case with a review of the literature.

Keywords: Meniscus abnormality, medial meniscus, conjoined double meniscus.

Copyright $\left({ }^{\circ} 2020\right.$ The Author(s): This is an open-access article distributed under the terms of the Creative Commons Attribution 4.0 International License (CC BY-NC 4.0) which permits unrestricted use, distribution, and reproduction in any medium for non-commercial use provided the original author and source are credited.

\section{INTRODUCTION}

Various shapes of congenital abnormalities of the menisci have been reported. Among them, the conjoined double meniscus is extremely uncommon. Anomalies of the menisci tend to affect the lateral meniscus more commonly and are reportedly more prevalent in Asians than Americans or European populations. There have been a few reports of doublelayered lateral meniscus. We report a case of congenital conjoined double medial meniscus which has never been reported yet.

\section{Patient And ObServations}

A 30-year-old male with a history of sports injury left knee8 years back. He presented feeling of instability and "giving way" during walking or running with mild pain. He had been experiencing mild pain and discomfort in the left knee for last 3 years. On physical examination, he had a positive anterior drawer test, Lachman test, Pivot shift test but no joint line tenderness and negative McMurray's test. There were no signs of locking, clicking in the left knee and no ligamentous laxities in other joints. He had normal gait, full range of motion but 'giving way' sensation. Neurovascular examination was normal. There was no bony anomaly. Clinically we diagnosed as old anterior cruciate ligament injury with osteoarthritic changes. Magnetic resonance images (MRI) was done but we could not diagnose the meniscus anomaly from MRI film. There was complete tear of anterior cruciate ligament (Fig. 1).On arthroscopy, old complete tear of anterior cruciate ligament was observed. Incidentally we found the medial meniscus had another meniscus overlying a normal one. The upper meniscus extended throughout the whole length and connected to lower meniscus through a junctional cleavage. Its periphery was connected to the joint capsule. The posterior portion of upper accessory meniscus was also connected to the joint capsule (Fig. 2). The accessory meniscus was not mobile on probing. So we think that it was not an origin of symptom from meniscus. Mild pain may be due to osteoarthritic changes. Only anterior cruciate ligament reconstruction was done. Conjoined double medial meniscus was not resected. MRI was reviewed after surgery. Postoperatively, ambulation with early weight bearing was permitted, and the preoperatively pain was alleviated. Three months after surgery, the patient could walk freely and returned to normal life without any discomfort.

\section{DISCUSSION}

Various anomalies of meniscus have been reported. Among those discoid meniscus was the most common anatomic variant of the meniscus [1]. Other less common anomalies include incomplete discoid meniscus, Wrisberg meniscus, ring-shaped meniscus, and accessory meniscus, congenital separation of meniscus, congenital partial deficient meniscus and absence of the menisci [2]. Double-layered meniscus have been reported with a prevalence of $0.06 \%$ to $0.09 \% .3$ ) Suzuki et al.[1] reported two cases of double- 

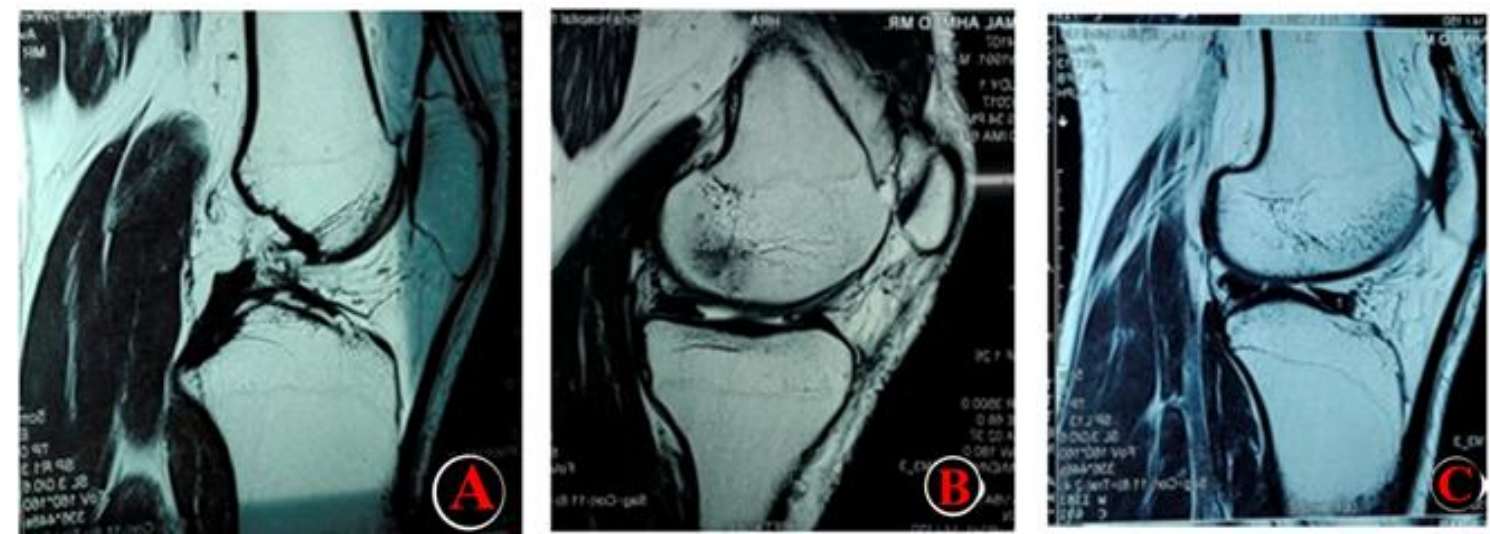

Fig-1: (A) Magnetic resonance imaging (MRI) showing Complete ACL tear. (B) And (C) MRI showing double meniscus
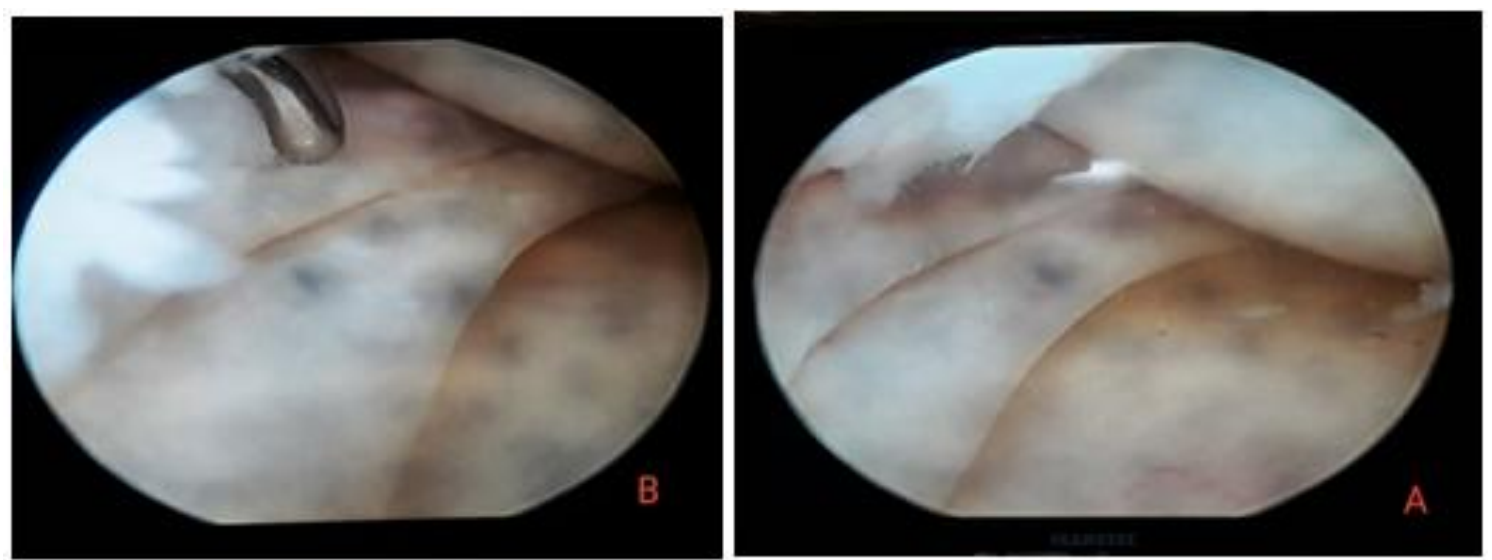

Fig-2: Arthroscopic findings. (A) An additional semicircular meniscus was observed over the normal medial meniscus. (B) The upper meniscus was attached to the lower meniscus throughout having a cleavage. Its periphery was connected to the joint capsule and the lower meniscus. Its posterior edge was attached to joint capsule

Double-layered medial meniscus is an extremely rare anatomical abnormality; it is more in lateral meniscus. Double-layered lateral meniscus was thin, mobile in the previous case report $[1,4]$. But in this case, double medial meniscus were conjoined and connected to each other. It was not mobile. We think that it was not the origin of pain. These findings were different from the double-layered lateral meniscus which was reported previously [1-3, 5]. Komatsu et al. [6] reported double-layered medial meniscus. The anterior portion of the medial meniscus had two layers, attached to the anterior surface of the tibia. In this case, the medial meniscus is double and it was attached to normal medial meniscus. Lee and Min [7] reported abnormal band of the lateral meniscus. It was characteristic that upper abnormal band was loose and serpentine. In this case accessory meniscus was neither loose, nor mobile. Regarding the differentiation of double layered meniscus from a horizontal tear, the cleavage of a horizontal tear is sharp margin, irregular in shape [3]. In this case, the margin was smooth, regular in both meniscuses. Both the meniscus was attached with each other throughout and it was the complete conjoined double medial meniscus. Probing was done to see the cleavage or tear but it was found fixed and attached. In addition, there was no problem with respect to the volume of the residual meniscus suggesting that the anomaly was not due to a horizontal tear (Fig. 2). Based on these findings, we concluded that this anomaly was different from acquired changes induced by degeneration or trauma. Menisci differentiate directly from blastemal cells connected to the capsule $[4,8]$. The causes of such variations are multifactorial, including congenital and developmental influences, but clinical presentations, pathology, and epidemiology of variations, altered biomechanics of medial compartment are still not clear [2]. After surgery, the patient had no discomfort or pain. We think that congenital conjoined double-layered medial meniscus was not the cause of pain. It is unclear how double-layered medial meniscus influences knee joint biomechanics and also double-layered medial meniscus can be a cause of meniscus tear. Congenital conjoined double-layered medial meniscus is an extremely rare anatomical abnormality. We thought that abnormal band of meniscus [7], double-layered meniscus [1-3], separated meniscus [6] were similar meniscus abnormalities. But they were a little different. It was unclear that morphological classification and its characteristics. It is expected to clarify its 
characteristics, altered biomechanics, influence to original meniscus and causes of congenital conjoined double medial meniscus.

\section{CONFLICTS OF INTEREST}

The authors have nothing to disclose.

\section{REFERENCES}

1. Suzuki S, Mita F, Ogishima H. Double-layered lateral meniscus: a newly found anomaly. Arthroscopy. 1991; 7:267-71.

2. Takayama K, Kuroda R, Matsumoto T. Bilateral double-layered lateral meniscus: a report of two cases. Knee Surg Sports Traumatol Arthrosc. 2009; 17:1336-9.
3. Okahashi K, Sugimoto K, Iwai M, Oshima M, Fujisawa Y, Takakura Y. Double-layered lateral meniscus. J Orthop Sci. 2005; 10:661-4

4. Karataglis D, Dramis A, Learmonth DJ. Doublelayered lateral meniscus. A rare anatomical aberration. Knee. 2006;13: 415-6.

5. Wang Q, Liu XM, Liu SB, Bai Y. Double-layered lateral meniscus. Knee Surg Sports Traumatol Arthrosc. 2011; 19:2050-1.

6. Komatsu N, Yamamoto K, Chosa E. Bilateral congenital separation of the lateral meniscus. A case report. Knee. 2008; 15:330-2.

7. Lee BI, Min KD. Abnormal band of the lateral meniscus of the knee. Arthroscopy. 2000; 16:11.

8. Hosea TM, Tria AJ Jr, Bechler JR. Evolution and embryology of the knee. In: Insall JN, Scott WN, ed. Surgery of the knee. 3rd ed. New York: Churchill-Livingstone; 2001: 3-12. 